\title{
Editorial
}

Martin R. Lessard MD FRCPC, Claude A. Trépanier MD FRCPC

\section{Use and misuse of} syringes in anaesthesia

\section{A} S early as 1873 , Skinner raised the possibility that anaesthetists and their equipment might be a possible vector for nosocomial infections. ${ }^{1}$ However, more than a century later, many anaesthetists still have working habits that are considered at risk for transmission of bloodborne pathogens between patients. Among these practices, the use of a common syringe to administer medication to more than one patient (termed hereafter: reuse of syringes) is very common. In a recent survey of members of the American Society of Anesthesiologists (ASA), Tait et al. reported that $47 \%$ of anaesthetists reuse syringes for more than one patient. ${ }^{2}$ Last year, a study by Kantor $e t$ al. reported a similar figure among Canadian anaesthetists (61\% reusing syringes). ${ }^{3}$ Given the large publicity about the AIDS epidemic and the risk of transmission of bloodborne pathogens, these findings are disturbing. Many studies have described the risks associated with the reuse of syringes. It has been shown that syringes can become contaminated with blood after being in contact with the injection ports of $i v$ tubings. ${ }^{4}$ The presence of a check valve in the $i v$ tubing does not prevent blood reflux. Morcover, blood contamination of injection ports frequently cannot be detected by visual inspection. ${ }^{4}$ Finally, it has also been clearly demonstrated that changing the needle was ineffective in preventing contamination of syringes and their contents. ${ }^{4}$ Warnings against the practice of reusing syringes have been published in the last ten years. ${ }^{5,6}$ Moreover, in 1992, the Committee on Occupational Health of Operating Room Personnel of the ASA published recommendations specifically addressing the practice of anaesthesia. ${ }^{7}$ It is surprising that the practice of reusing syringes is still so prevalent. Concern about cost containment may be one reason, as might also be the cumbersome task of preparing a new set of syringes for each case and the reluctance to change convenient working habits. Ignorance of both the risks associated with the practice of reusing syringes and the infection control recommendations are probably involved too.
Using data from the literature, a fairly accurate estimate of the risk of transmission of bloodborne infections associated with the reuse of syringes in anaesthesia can be made. In anaesthesia, most of the time, syringes are used to administer medications into injection ports or three-way stopcocks of iv tubing that might be contaminated with the patient's blood. That situation differs from direct intravenous or subcutaneous injections where an unequivocal contact with the patient's body fluids is made. Thereby, in the anaesthetic setting, the risk of cross-infection is determined by three factors: first, the risk of blood contamination of a syringe after making contact with the injection port of the iv tubing; second, the prevalence of the infection in the general population and most specifically in the surgical population; and third, the risk of seroconversion after exposure to contaminated blood. In a previous study we estimated that the risk of blood contamination of a syringe after contact with the injection port of an $i v$ infusion ranges from as low as $0 \%\left(\mathrm{CI}_{95}=0.00-1.22\right)$ to as high as $3.33 \%\left(\mathrm{CI}_{95}=2.26-4.73\right)$ depending mainly on the distance of the injection port from the $i v$ catheter. $^{4}$ These numbers were obtained from a sample of $i v$ lines started in the operating room. Many factors can greatly increase the rate of blood contamination of $i p$ tubings: "old" iv lines started on the ward before surgery, noninvasive blood pressure cuff on the same arm as the iv catheter, infusion of blood products into the $i v$ line. Most recent data estimate the prevalence of human immunodeficiency virus (HIV) infection in North America at $0.3 \%{ }^{8}$ However, a higher prevalence rate $(0.4 \%)$ has been reported among elective surgical patients. ${ }^{9}$ Furthermore, a Canadian hospital reported that $0.6 \%$ of patients admitted were HIV-positive, ${ }^{10}$ and much higher rates are reported in some United States hospitals. ${ }^{11}$ The prevalence rate of hepatitis B (HBV) infection and of hepatitis $\mathrm{C}(\mathrm{HCV})$ infection in Canada are estimated at $0.5 \%$ and $0.7 \%$ respectively. ${ }^{12,13}$ Here also, prevalence rates are probably higher among patients than in the general population. Finally, the 
seroconversion rates after parenteral exposure to blood contaminated with $\mathrm{HIV}, \mathrm{HBV}$ or $\mathrm{HCV}$ are estimated at $0.3 \%, 5 \%$ (up to $30 \%$ if the carrier is $\mathrm{HBeAg}$ positive), and $10 \%$ respectively. ${ }^{14}$ These numbers are taken from data from accidental parenteral exposure of healthcare workers (for obvious reasons, the only data available). This setting is different from a direct iv injection which might carry a different risk. Combining all these data, the risk of transmission of bloodborne infection associated with the practice of reusing syringes in anaesthesia can be calculated as follow:

Risk $=$ syringe contamination rate $\times$

prevalence rate $x$ seroconversion rate

Therefore the estimated risk of transmission of HIV is anywhere between 0 and $1 / 9,000,000$ using the lowest figures, increasing up to $1 / 1,000,000$ when using the highests. Using the same calculation method, the estimates for $\mathrm{HBV}$ and $\mathrm{HCV}$ range between 0 and $1 / 328,000$ (up to $1 / 14,000$ with the highest figures) for $\mathrm{HBV}$, and between 0 and $1 / 117,000$ (up to $1 / 30,000$ with the highest figures) for HCV. It must be remembered that these figures can be increased markedly in area of higher prevalence of these infections as well as by careless working habits.

Transmissions between patients of bloodborne pathogens associated with the reuse of syringes, which is actually more worrying than the risks of cross-infection calculated above, have been recently reported. In 1993 the New South Wales Health Department initiated an investigation that led to the conclusions that four patients had been infected with HIV on the same day by cross contamination from a fifth HIV-positive patient in a private surgical clinic. ${ }^{15}$ The most likely mode of transmission was a break in the infection control practices such as the reuse of a vial or a syringe of local anaesthetic. ${ }^{16}$ Even more worrying is the outbreak of hepatitis B (HBV) in patients undergoing electroconvulsive therapy (ECT) ${ }^{17}$ During the same week, six patients were infected with $\mathrm{HBV}$ from another carrier patient ( $\mathrm{HBsAg}$ and $\mathrm{HBeAg}$ positive). The same syringes were attached to IV stopcocks to administer the anaesthetic medications and were reused for all patients. Finally, the investigations of several outbreaks of severe postoperative bacterial infections related to the contamination of propofol have also implicated the reuse of syringes and infusion-pump lines as one of the risk factors. ${ }^{18}$ Obviously, these few reports grossly underestimate the magnitude of the problem since $\mathrm{HIV}, \mathrm{HBV}$ and $\mathrm{HCV}$ infections usually cannot be easily diagnosed in the postoperative period. Especially for HIV, the long delay before diagnosis is made makes it difficult to associate the infection with the anaesthetic experience. However should an outbreak emerge, Public Health Departments have shown their ability to easily identify the source of the infection. . $^{15,17,18}$

In 1987, the Center for Disease Control of the United States (CDC) published Recommendations for Prevention of HIV Transmission in Health-Care Settings. ${ }^{19}$ In these guidelines, the "Universal Precautions" require that blood and blood-contaminated body fluids of all patients be considered as potentially infectious. However, these recommendations and their 1988 and 1991 updates did not specifically address the issue of the reuse of syringes in an anaesthetic setting. In 1992 the ASA published Recommendations for Infection Control for the Practice of Anesthesiology. ${ }^{7}$ These guidelines are very clear: "Medication from a syringe must not be administered to multiple patients even if the needle on the syringe is changed. After entry into or connection with a patient's intravenous infusion, the syringe and needle should be considered contaminated...." 7 Obviously, a used syringe should never be used to draw medication from a multidose vial either. The same recommendations apply to drug infusions (fluids, containers and tubings) which are single patient-use. In 1995 the Anesthesia Patient Safety Foundation (APSF) made the same recommendations but with the addition of even more stringent precautions for syringes and infusions containing propofol. ${ }^{20}$ Unfortunately, these recommendations (especially those of the APSF) have not been widely promoted among Canadian anaesthetists. In Canada, only the Laboratory Centre for Disease Control (Health Protection Branch, Health Canada) has briefly addressed the problem in the Infection Control Guidelines: Preventing the Transmission of Bloodborne Pathogens in Health Care and Publi Service Settings. 21

It must be recognized that discarding all used syringes and needles after each case could result in a considerable waste of anaesthetic drugs. However, by modifying working habits, wastage can be reduced to a minimum. Only the exact dose of drug that will be administered to the patient should be drawn into a syringe. The use of medication in small volume ampullae and vials, or in multidose vials (with a cautious aseptic technique) can also be cost effective ways to minimize drug wasting. However, the practice of reusing syringes can never be justified by cost containment objectives.

In summary, although the estimated risk of transmission of bloodborne pathogens associated with the practice of reusing syringes in anaesthesia is low, it is real and not trivial. This risk can be markedly increased by the anaesthetists' working habits and by the local prevalence of the infections. Cases of transmission of bloodborne pathogens have been reported and several other 
cases may have gone unrecognized. For all theses reasons, every anaesthetist should adhere to the current recommendations. The practice of reusing a syringe to administer medication to more than one patient is unacceptable and must be totally abandoned.

\section{Les seringues en anesthésie : bons et mauvais usages}

Dès 1873, Skinner a évoqué la possibilité que l'anesthésiste et son équipement puissent être un vecteur pour la transmission d'infections nosocomiales. ${ }^{1}$ Cependant, plus d'un siècle plus tard, plusieurs anesthésistes ont encore des habitudes de travail qui peuvent être considérées comme étant un risque pour la propagation entre patients d'infections transmissibles par le sang. Parmi ces pratiques, l'emploi d'une seringue commune pour l'administration d'un médicament à plus d'un patient (nommé ci-après: réutilisation des seringues) est encore très fréquent en anesthésie. Dans un récent sondage auprès des membres de l'American Society of Anesthesiologists (ASA), Tait et al. ont rapporté que $47 \%$ des anesthésistes réutilisent leurs seringues pour plus d'un patient. ${ }^{2}$ Dans une étude publiée l'an dernier, Kantor et al. ont observé une pratique comparable parmi les anesthésistes canadiens (i.e., $61 \%$ réutilisent les seringues). ${ }^{3}$ Considérant la vaste publicité faite à l'épidémie du SIDA et le risque de propagation de pathogènes transmissibles par le sang, ces chiffres sont plutôt inquiétants. Plusieurs études ont décrit les risques associés à la réutilisation des seringues. Il a été démontré que les seringues peuvent être contaminées avec du sang après avoir été en contact avec les sites d'injection des tubulures de perfusions intraveineuses (tubulures IV). ${ }^{4} \mathrm{De}$ plus, la présence d'une valve anti-reflux sur la tubulure IV ne prévient pas la contamination par le sang des sites d'injection. La plupart du temps, la contamination des sites d'injection n'est pas visible à l'oeil nu. ${ }^{4}$ Finalement, il a aussi été clairement démontré que changer uniquement l'aiguille n'est pas efficace pour prévenir la contamination des seringues et de leur contenu. ${ }^{4}$ Des mises en garde contre la pratique de réutilisation des seringues ont été publiées durant les dix dernières années. ${ }^{5,6}$ De plus, en 1992, le Comité de l'ASA sur la santé au travail du personnel de salle d'opération a publié des recommandations spécifiques à la pratique de l'anesthésie. ${ }^{7}$ Il est donc surprenant de voir que la réutilisation des seringues soit encore si répandue. Plusieurs raisons peuvent être évoquées: le contrôle des coûts des médicaments, l'augmentation de la charge de travail pour la préparation de nouvelles seringues pour tous les cas, et la résistance à modifier des habitudes de travail solidement implantées. L'ignorance des risques associés avec la pratique de la réutilisation des seringues et des recommandations pour le contrôle de l'infection sont probablement aussi des facteurs impliqués.

En utilisant des données de la littérature, on peut calculer assez précisément le risque de propagation des infections transmissibles par le sang associé à la réutilisation des seringues. En anesthésie, la plupart du temps, les seringues sont employées pour administrer des médicaments via les sites d'injection ou les robinets 3-voies des tubulures IV qui peuvent être contaminés par le sang du patient. Cette situation est différente d'une injection sous-cutanée ou intraveineuse directe où il y a un contact non équivoque avec des liquides biologiques du patient. En anesthésie, le risque d'infection croisée est donc dépendant de trois facteurs : premièrement le risque de contamination par le sang d'une seringue qui entre en contact avec le site d'injection d'une tubulure $I V$, deuxièmement la prévalence de cette infection dans la population générale et plus spécifiquement dans la population chirurgicale, et troisièmement le risque de séroconversion après une exposition à du sang contaminé. Dans une étude antérieure, nous avons estimé que le risque de contamination sanguine d'une seringue après contact avec le site d'injection d'une tubulure IV variait de $0 \%\left(\mathrm{CI}_{95 \%}=0.00-1.22\right)$ jusqu'à $3.33 \%\left(\mathrm{CI}_{95 \%}=2.26-4.73\right)$, dépendant de la distance entre le site d'injection et le cathéter intraveineux. ${ }^{4}$ Ces chiffres ont été obtenus à partir d'un échantillonnage de tubulures IV installées en salle d'opération. Plusieurs facteurs peuvent grandement augmenter la fréquence de contamination par le sang des tubulures IV: perfusion intraveineuse débutée dans une unité bien avant la chirurgie, brassard à pression artérielle non invasive installé sur le même bras que le cathéter intraveineux, administration de produits sanguins par la tubulure IV. La prévalence de l'infection par le virus de l'immunodéficience humaine (VIH) en Amérique du Nord est estimée à $0.3 \%{ }^{8}$ Cependant, un plus haut taux de prévalence $(0.4 \%)$ a été rapporté parmi une population chirurgicale élective. ${ }^{9}$ De plus, un hôpital canadien a rapporté que $0.6 \%$ des patients admis étaient séropositifs. ${ }^{10}$ Des taux encore plus élevés ont été rapportés dans certains hôpitaux américains. ${ }^{11}$ Les taux de prévalence des infections par le virus de l'hépatite $B$ (VHB) et de 
l'hépatite $\mathrm{C}(\mathrm{VHC})$ au Canada sont estimés à $0.5 \%$ et $0.7 \%$ respectivement. ${ }^{12,13}$ Encore une fois, la prévalence est probablement plus élevée parmi les patients que dans la population générale. Finalement, les taux de séroconversion après une exposition parentérale à du sang contaminé avec le VIH, VHB et VHC sont estimés à $0.3 \%, 5 \%$ (pouvant atteindre $30 \%$ si le porteur est $\mathrm{AgHBe}$ positif) et $10 \%$ respectivement. ${ }^{14}$ Notons que ces chiffres proviennent de données obtenues suite à des expositions parentérales accidentelles de travailleurs de la santé (pour des raisons évidentes, les seules données disponibles). Ce contexte est différent d'une injection intraveineuse directe qui pourrait être associée à un risque différent de séroconversion. En combinant toutes ces données, le risque de propagation entre patients d'une infection transmissible par le sang associé avec la pratique de réutilisation des seringues en anesthésie peut être calculé comme suit:

Risque = taux de contamination des seringues * taux de prévalence * taux de séroconversion

Donc, le risque estimé de transmission du VIH est quelque part entre 0 et $1 / 9,000,000$ en employant les chiffres les plus faibles, pouvant atteindre $1 / 1,000,000$ avec les chiffres les plus élevés. De la même façon, le risque pour le VHB et le VHC peut être estimé entre 0 et $1 / 328,000$ (jusqu'à $1 / 14,000$ avec les chiffres les plus élevés) pour le VHB, et entre 0 et $1 / 117,000$ (jusqu'à $1 / 30,000$ avec les chiffres les plus élevés) pour le VHC. Il faut rappeler que ces risques peuvent être grandement augmentés dans les zones à haute prévalence pour ces infections et par des habitudes de travail négligentes.

Des cas de transmission d'infections associés avec la réutilisation des seringues ont récemment été rapportés, ce qui est encore plus inquiétant que les risques calculés ci-haut. En 1993, le département de santé publique du New South Wales a mené une enquête qui a conduit à la conclusion que quatre patients avaient été infectés avec le VIH dans une clinique chirurgicale privée au cours d'une même journée, par une contamination croisée à partir d'un cinquième patient séropositif. ${ }^{15}$ Le mode de transmission le plus probable était une faille dans les mesures de prévention de l'infection telle la réutilisation d'un vial multidose ou d'une seringue d'anesthésique local. ${ }^{16}$ Une épidémie d'hépatite $B$ chez des patients ayant subi des électrochocs est encore plus inquiétante. ${ }^{17}$ Durant la même semaine, six patients ont été infectés par le VHB à partir d'un autre patient porteur (AgHBs et $\mathrm{AgHBe}$ positif). Les mêmes seringues étaient utilisées pour administrer les médicaments anesthésiques via les robinets 3-voies des tubulures IV à tous les patients. Enfin, les enquêtes portant sur plusieurs séries d'infec- tions bactériennes postopératoires graves reliées à la contamination du propofol ont aussi impliqué la réutilisation des seringues et des tubulures de pompe à perfusion parmi les facteurs de risque identifiés. ${ }^{18}$ De toute évidence, ces quelques cas sous-estiment grossièrement l'importance du problème puisque les infections à VIH, VHB et VHC sont rarement diagnostiquées dans la période postopératoire immédiate. Particulièrement pour le VIH, un très long délai avant le diagnostic rend difficile l'association d'une infection avec la technique anesthésique. Cependant, advenant une série de cas, les départements de santé publique ont démontré leur capacité à identifier facilement la source de l'infection. ${ }^{15,17,18}$

En 1987, le Center for Disease Control des ÉtatsUnis (CDC) a publié Recommendations for Prevention of HIV Transmission in Health-Care Settings. ${ }^{19}$ Dans ce document, les "Précautions Universelles" requièrent que le sang et les liquides biologiques de tous les patients soient considérés comme potentiellement infectieux. Cependant, ces recommandations et leur mise à jour de 1988 et 1991 ne s'adressaient pas spécifiquement au problème de réutilisation des seringues dans le contexte anesthésique. En 1992, l'ASA a publié Recommendations for Infection Control for the Practice of Anesthesiology.? Ces recommandations sont très claires : «Un médicament contenu dans une seringue ne doit pas être administré à plus d'un patient, même si l'aiguille est changée. Dès qu'elles ont été en contact avec la perfusion intraveineuse d'un patient, la seringue et l'aiguille devraient être considérées contaminées . . . »? ${ }^{7}$ Bien entendu, une seringue usagée ne devrait jamais être utilisée pour prélever un médicament d'un vial multidose. Les mêmes recommandations s'appliquent pour les perfusions de médicaments (liquide, contenant et tubulure) qui doivent être à l'usage d'un seul patient. En 1995, l'Anesthesia Patient Safety Foundation (APSF) a fait les mêmes recommandations mais avec l'ajout de précautions encore plus strictes pour les seringues et les perfusions de propofol. ${ }^{20}$ Malheureusement, ces recommandations (particulièrement celles de l'APSF) n'ont pas été largement diffusées auprès des anesthésistes canadiens. Au Canada, il n'y a que le Laboratoire de Lutte Contre la Maladie (Direction Générale de la Protection de la Santé, Santé Canada) qui fait brièvement mention du problème dans le Guide de prépention des infections transmissibles par le sang dans les établissements desanté et les services publics. ${ }^{21}$

Il faut cependant reconnaitre que jeter toutes les seringues utilisées après chaque cas peut amener un gaspillage important des médicaments anesthésiques. Cependant, en modifiant ses habitudes de travail, ce gaspillage peut être considérablement réduit. Ainsi, seulement la quantité exacte de médicament à admi- 
nistrer au patient devrait être aspirée dans la seringue. L'emploi de médicaments dans des vials et ampoules de petit volume ou l'emploi de vials multidoses (avec une technique aseptique stricte) peut aussi être une façon efficace de diminuer le gaspillage de médicaments. De toute façon, le contrôle des dépenses de médicaments ne peut d'aucune façon justifier la réutilisation des seringues.

En résumé, même si le risque de propagation d'une infection transmissible par le sang associé à la pratique de réutilisation des seringues en anesthésie est faible, il est réel et non négligeable. Ce risque peut être grandement augmenté par les habitudes de travail d'un anesthésiste et par la prévalence locale de ces infections. Des cas de transmission d'infections ont été rapportés et plusieurs autres sont probablement passés inaperçus. Pour toutes ces raisons, les anesthésistes n'ont d'autre choix que de se conformer aux recommandations de prévention des infections. L'utilisation d'une même seringue pour administrer des médicaments à plus d'un patient est inacceptable et doit être totalement abandonnée.

\section{References}

1. Skinner. Anaesthetics and inhalers.(Letter) BMJ 1873: 1: 353-4.

2 Tait $A R$, Tuttle $D B$. Preventing perioperative transmission of infection: a survey of anesthesiology practice. Anesth Analg 1995; 80 : 764-9.

3 Kantor GSA, Chung $F$. Anaesthesia drug cost, control and utilization in Canada. Can J Anaesth 1996; 43: 9-16.

4 Trépanier CA, Lessard MR, Brochu JG, Denault PH. Risk of cross-infection related to the multiple use of disposable syringes. Can J Anaesth 1990; 37: 156-9.

5 Heseltine P. Anesthesiologists should not give IV medications with common syringe (Letter). Hospital Infection Control 1986; $13: 84-5$.

6 Browne RA, Chernesky $M A$. Infectious diseases and the anaesthetist. Can J Anaesth 1988; 35: 655-65.

7 American Society of Anesthesiologists. Committee on Occupational Health of Operating Room Personnel. Recommendations for Infection Control for the Practice of Anesthesiology. Park Ridge, Ill: American Socicty of Anesthesiologists, 1992.

8 WHO. Weekly Epidemiological Record, 1994; 69: 5-12.

9 Charache P, Cameron JL, Maters AW, Frantz EI. Prevalence of infection with human immunodeficiency virus in elecrive surgery patients. Ann Surg 1991; 214: 562-8.

10 Louie $M$, Low DE, Feinman SV, McLaughlin B, Simor $A E$. Prevalence of bloodborne infective agents among people admitted to a Canadian hospital. Can Med Assoc J 1992; 146: 1331-4.

11 Janssen RS, St. Louis ME, Satten GA, et al. HIV infection among patients in U.S. acute care hospitals. Strategies for the counseling and testing of hospital patients. N Engl J Med 1992; 327: 445-52.

12 Varughese $P$. Hepatitis B infection in Canada. Canada Diseases Weekly Report 1985; 11: 1-4.

13 Murphy DG, Willems B, Delage $G$, et al. Hepatitis C virus genotypes in patients and blood donors Québec. Canada Communicable Disease Report 1995; 21: 129-32.

14 Lanphear BP. Trends and patterns in the transmission of bloodborne pathogens to health care workers. Epidemiol Rev 1994; 16: 437-50.

15 Chant K, Lowe D, Rubin G, et al. Patient-to-patient transmission of HIV in private surgical consulting rooms (Letter). Lancet 1993; 342: 1548-9.

16 Shields JW. Patient-to-parient transmission of HIV (Letter). Lancet 1994; 343: 415.

17 Froggatt JW, Dover DM, Stephens MA. Hospital outbreak of hepatitis $B$ in patients undergoing electroconvulsive therapy (Abstract). Program and Abstracts of the 31 st Interscience Conference on Antimicrobial Agents and Chemotherapy. Chicago, September 29-October 2, $1991 ; \mathrm{A} 347$.

18 Bennett $S N$, McNeil MM, Bland LA, et al. Postoperative infections traced to contamination of an intravenous anesthetic, propofol. N Engl J Med 1995; 333: $147-54$

19 Center for Disease Control. Recommendations for prevention of HIV transmission in health-care settings. Morbidity and Mortality Weekly Report 1987; 36: 2S-18.

20 Berry AJ. Recommendations for handling parenteral medications used for anesthesia or sedation. Pittsburgh: Anesthesia Patient Safety Foundation, 1995.

21 Laboratory Centre for Disease Control. Infection control guidelines. Preventing the transmission of bloodborne pathogens in health care and public service settings. Canada Communicable Disease Report 1997; $23(53)$. 